\title{
Aerobics Teaching Research in View of Overweight Students
}

\author{
Xiao-yan REN ${ }^{1, a,{ }^{*}}$ \\ ${ }^{1}$ Henan Quality Polytechnic, Pingdingshan, Henan, China \\ aspring.gump@163.com
}

Keywords: Aerobics, Teaching, Overweight students.

\begin{abstract}
Aerobics has become an important content of quality education and aesthetic education in colleges and universities because of its unique charm of sports. But in aerobics class a small number of overweight and obese students doesn't get enough attention, this part of students are often marginalized. Overweight students have physical and psychological difficulties, we have this two problems analyzed. Based on the analysis, using scientific teaching methods, mainly by improving the overweight students' classroom participation, rationally thinking of overweight students' physical characteristics and movement difficulties, easing overweight students by psychological counseling, make the overweight students better blend into class, thus improve learning effect.
\end{abstract}

\section{Introduction}

Aerobics is the combination of gymnastics, dance and music. It is a set of gymnastics and dance movement composed according to the requirements of the comprehensive coordinated development of the body and is performed in the music accompaniment. It is a means of exercise to achieve better health, cultivate correct posture, shape the form of beauty, and edify sentiment of beauty [1].

With its unique charm of sports and art, aerobics has a broad mass base of the crowd, and has been an important content of quality education and aesthetic education. With the change of people ideas and the continuous development of quality education reform in recent years, the teaching of physical education in colleges is gaining more and more attention. As one of the most favorite sports aerobics also get fast development.

Aerobics has the function of reducing fat, improving physical beauty, body and quality [2]. Aerobics movements are abundant and changeable, they need coordination and effort of the whole body in music accompaniment to finish the action. A variety of fast strong, sharp action can not only enhance the explosive force of muscle, but also improve the body's muscles' coordinate ability, and conversion ability between motorial excitement and cerebral cortex inhibition. Long time (more than 10 minutes) exercise can improve the body's aerobic capacity, reduce the accumulation of lactic acid in the motion, make the body not easy to feel tired, thus enhances the body's endurance. In form practice, some soft movement and a variety of dance movements can enhance the muscle strength and muscle ligament flexibility and elasticity, enhance the coordination of the body. Mat exercises mainly enhanced lumbar muscles, chest and back muscle and leg muscle strength, and eliminate the excess fat. More than enough to illustrate aerobics exercise on body quality is comprehensive and the influence of the various physical quality can be significantly improved [3].

Aerobics has obvious effect to improve students' physical fitness, and it is a physical exercise for overweight students. Basically every aerobics class has some overweight students. Because of small proportion, they have not received the teacher's attention. 
Together with these overweight students physical and psychological difficulties, this part of the students are often marginalized in aerobics class. So that the learning effect is not good and it is difficult to attain the goal of learning.

In the existing studies, there are a number of researches on the fitness function for the fat of aerobics, a number of studies of aerobics teaching, and some researches on how to help overweight students for physical education. These research interests are mainly concentrated in obese students' physical and mental health problems, aerobics fitness function and aerobics teaching. Due to the amount and proportion of overweight students in class is small, problems of overweight students didn't cause enough attention to teachers and researchers. Yet till now there are no specialized researches on overweight students in aerobics class.

Studies have shown that overweight students have a strong sense of weight loss and fitness, they hope to improve themselves through sport like aerobics [4]. But overweight students have difficulties in learning aerobics, which mainly display in two aspects, physical and psychological. In the physical factors, here we mainly discuss how to better help overweight students overcome learning difficulties in class teaching, so as to achieve a satisfactory effect. In the psychological factors, discuss how to help overweight students overcome psychological problems from the psychology Angle.

\section{Physical Factors Analysis}

In recent years, with the improvement of people's material life, the incidence of obesity of college students in our country increased year by year, obesity has become a problem that does not allow ignoring. At present, the designated standard about obesity in college students is the adults BMI standard in Asia recommended by the world health organization. The overweight and obesity standard in Asia is as follows: BMI $\geq 23 \mathrm{~kg} / \mathrm{m}^{2}$ for overweight. Among them, the BMI in $23 \sim 30 \mathrm{~kg} / \mathrm{m}^{2}$ for obesity.

\section{Poor Physical Quality}

Sports standard test data show that in normal weight, overweight and obesity college students with the increasing of the weight level the physical qualities gradually reduce, namely the fatter the students, the worse the sports scores are. Overweight students' endurance running performance, vital capacity are lower than the normal weight students'. And the index of heart rate, blood pressure, and the steps index are significantly higher than normal-weight students, this shows lower cardiopulmonary functions of overweight students.

\section{Poor Muscle Strength}

Overweight students' muscle strength is poor than normal students. This is because on the one hand muscle exercise is less at ordinary times, on the other hand, more energy is consumed to remove excess fat when muscle actives, so that affects the whole muscles work output. Adverse impact of obesity on its performance is particularly prominent in activities.

\section{Nonstandard Actions}

Due to obese body, overweight students' movements are not flexible. Especially for aerobics movements, they are hardly standard. This cause frustration to overweight students. 


\section{Psychological Factors Analysis}

Due to obesity problem, overweight college students face significantly higher psychological pressure than that of normal-weight students. They are high-risk groups of mental health problems. They have psychological health problems in daily life, relationships and emotions.

\section{Anxiety and Depression}

Overweight college students care much about others opinion of himself, with a higher level of implicit self-esteem. Depression and anxiety are prominent symptoms of obesity students, they prone to derive depression syndrome, such as anxiety, depression. Those who feel they are obese tend to take more psychological pressure. [5]

\section{Higher Interpersonal Sensitivity}

Behavioral problems of obese students mainly displays in daring not to speak in front of people, small communication range, easy to be shy or timid. Obese students are often made fun of or rejected by other students, which makes interpersonal relationship problems such as hostility and withdrawn. It shows that obese students showed higher interpersonal sensitivity, more care about their physical condition evaluation.

\section{Low Self-confidence and Self-esteem}

Self-confidence level will affect individual learning, competition, employment, and achievements, and many other aspects of the psychology and behavior. Different self-esteem levels have different individual behavior expressions. Low self-esteem individuals tend to be more negative associated with anxiety, depression and other negative emotions. Studies found that obese students are worse than normal-weight students in such aspects as self-confidence, self-evaluation. The explicit physical self-esteem of obese college students is lower than normal-weight college students, obese college students appears lack of self-confidence, with a feeling of being isolation and humiliation.

\section{Teaching Method}

Based on the above analysis of the physical factors and psychological factors, using scientific teaching methods, mainly by improving the overweight students' classroom participation, rationally thinking of overweight students' physical characteristics and movement difficulties, easing overweight students by psychological counseling, make the overweight students better blend into classroom teaching, thus improve the overweight students learning effect.

\section{Promote Equal Cooperation of the Overweight Students with Other Students through "Team Learning" Teaching Method}

"Team learning" [6] teaching method, refers to under the guidance of teachers, given a certain amount of time and space, let the student organize the learning process. In the process of "team learning", encourage other students to treat the overweight students equally, make the overweight students in group activities enjoy the fun of friendship, prompt students to teach each other, assist each other and promote each other. In the act of cooperation and competition experience the fun, cultivate the students' learning ability and cooperation spirit. "Team learning" teaching method can make the overweight students feel the warmth of the collective in group activities and the force of 
unity. When using the "team learning" reasonable grouping is noticeable, as well as to strengthen the teachers' leading role and put an end to group for overweight unfair treatment.

\section{Improve Overweight Students' Participation through the Cooperation Teaching Method}

Cooperative teaching is a kind of participatory and collaborative teaching method, the teaching method take the student as the center, with flexible and intuitive teaching methods, encouraged students to participate in teaching, strengthen the information communication and feedback between teachers and students, so that the students can deeply understand and master knowledge, and to apply knowledge in practice. Cooperation teaching method is to learn with the cooperation, solidarity, mutual help of team members, to strengthen team member's interest in learning and effect through mutual evaluation among groups, internal evaluation in the group and competition among groups, and to make the learning resources more equal, and the motivation of learning more strong.

\section{Carry on Differentiated Teaching to Overweight Students through the Stratified Teaching Method}

Within a class, according to the students' learning conditions and the physiological and psychological characteristics, divide the students into groups. Make similar qualities of students into one group. Dynamically adjust groups according to the actual situation of students in a timely manner. design varied teaching goal for each layer of group, put forward varied learning requirements, offer varied counseling, carry on varied intensive training and assessment, so that each layer of students can gains by varied teaching means and methods. Based on the factor of the students' cognitive level, learning ability and own quality differences. "Stratified teaching" is implemented by teachers to complement each other, activate the students' inner study interest, improve the learning confidence, realize the students all-round development and common progress, realize the goal of learning without any students leaving behind.

\section{Design Overweight Students' Classroom Motion Parameters through the Exercise Prescription Method}

Exercise prescription has gradually become a science, is thought to be one of the most effective means of improving and enhancing physical fitness and promoting the health level. To design the exercise prescription of overweight students in aerobics class, can make their movement more scientific. Here the main object of the exercise prescription is the overweight students, the exercise content set to be aerobics sports.

Set exercise prescription parameter. The reasons for the obesity is various and comprehensive. But the most fundamental one is that the accumulation of heat in the body is more than the consumption. Therefore, the main goal of the exercise prescription is negative growth of heat. Among them, the movement is mainly responsible for the heat consumption. 1) The exercise intensity. Take heart rate as the control index of the exercise intensity. As the main object is overweight students, set the main part of the heart rate range of a movement in the 120-150 times/min. 2) Exercise time. According to the physiological characteristics of the oxidation of fat cells, in order to obtain high efficiency of the total quantity of heat consumption, set exercise time to 90 min. 3) Exercise frequency. Based on the characteristics of benign physiological adaptation, overweight students' class time is not less than 3 months, so that can get 
ideal result. According to the scheduling, the applied prescription will be set to 18 weeks.

\section{Stimulate the Overweight Students' Participation Enthusiasm by Appropriately Decrease the Teaching Requests and Discriminately Change the Method of Assessment Mode}

Because of body factor, overweight students hardly passed the physical examination, so they are afraid of physical examination, and even produce a psychological aversion to physical education [7]. In order to obtain satisfied teaching effect, the teacher can change the previous evaluation of "one size fits all" approach by improve the assessment mode to inspire the overweight students' participation enthusiasm and improve their test scores. It tend to get a good result. Such as: adopt "performance improvement" assessment method to grade the overweight students. That is, give several tests to overweight students in the middle of the course, after a certain time of exercise, test again, compare the size of its improvement, assess the learning effects according to the level of amplitude in the improvement, and then give the remarks. This assessment method is different from that of normal-weight students. In the teaching process, the adoption of this assessment method makes the overweight students be convinced that as long as through their own efforts they can improve their performance, and thus can reach the standard to pass the exam or to be good. This greatly mobilized the overweight students in the aerobics.

\section{References}

[1] Chun-lai Xiao. Aerobics [M]. Beijing: People's physical education Press, 2009.

[2] Jason L. Talanian, et al. Two weeks of high-intensity aerobic interval training increases the capacity for fat oxidation during exercise in women [J]. J Appl Physiol 102: $1439-1447,2007$.

[3] Rui-hua Lian, Xiao-fang Gong. Experimental research of aerobics can improve the obese female college students' physical function and quality [J]. Liaoning 2009, 24(1):49-50.

[4] Nicola Guess. A qualitative investigation of attitudes towards aerobic and resistance exercise amongst overweight and obese individuals [J]. Guess BMC Research Notes 2012, 5:191

[5] Bin Xie, Chun-hong Liu, Chou Chihping, et al. Weight perception and psychological factors in Chinese adolescents [J]. Journal of adolescent health, 2003, 33:202-210

[6] Juan-juan Feng. The experiment that the happy teaching method uses in universities and colleges' aerobics teaching of the professorship is studied [D]. Beijing: Beijing Sport University, 2008

[7] Jin-feng Zhao. Experimental research of Aerobics exercise on obese female college students' body self-esteem and mood effects [J]. Journal of Changsha University, 2010, 24(2):127-129 